\title{
Insights into the dynamics of SARS-CoV-2 pandemicvia Shannon-Fisher causality plane
}

\section{Leonardo H.S. Fernandes ( $\square$ leonardo.henrique@ufrpe.br)}

Department of Economics and Informatics, Federal Rural University of Pernambuco, Campus Serra

Talhada, PE 56909-535 Brazil https://orcid.org/0000-0001-8739-7511

\section{Fernando H.A. de Araújo}

Department of Statistics and Informatics, Federal Rural University of Pernambuco, Recife, PE 52171-900 Brazil https://orcid.org/0000-0001-5417-0513

\section{Maria A.R. Silva}

Federal Institute of Education, Science and Technology of Para'iba, Campus Cabedelo, PB 58103-772 Brazil https://orcid.org/0000-0002-7363-988X

\section{Research Article}

Keywords: SARS-CoV-2, COVID-19, Shannon-Fisher causality plane

Posted Date: June 22nd, 2020

DOI: https://doi.org/10.21203/rs.3.rs-36581/v1

License: (c) (1) This work is licensed under a Creative Commons Attribution 4.0 International License.

Read Full License 


\title{
Insights into the dynamics of SARS-CoV-2 pandemic via Shannon-Fisher causality plane
}

\author{
Leonardo H. S. Fernandes ${ }^{1, *}$, Fernando H. A. de Araújo ${ }^{2}$, and Maria A. R. Silva ${ }^{3}$ \\ ${ }^{1}$ Department of Economics and Informatics, Federal Rural University of Pernambuco, Campus Serra Talhada, PE \\ 56909-535 Brazil \\ ${ }^{2}$ Department of Statistics and Informatics, Federal Rural University of Pernambuco, Recife, PE 52171-900 Brazil \\ ${ }^{3}$ Departament of Biology, Federal Institute of Education, Science and Technology of Paraíba, Campus Cabedelo, \\ PB 58103-772 Brazil \\ *leonardo.henrique@ufrpe.br
}

\section{ABSTRACT}

This paper performs a systematic investigation into the temporal evolution of severe acute respiratory disease coronavirus 2 (SARS-CoV-2) pandemic considering 15 diverse countries. Based on the foundations of Information Theory, we apply the Shannon-Fisher causality plane (SFCP), to map the dynamics behavior inherent to SARS-CoV-2 and their respective locations along the (SFCP). Our results show that this dynamics varies widely along the SFCP from the lower-right region, characterized by high entropy and low degree of reliability in relation to the information extracted from the analyzed data set to the top-right region, characterized by the less entropic and high degree of reliability in relation to the information extracted from the analyzed data set. It reveals that we have three different groups of countries in controlling the SARS-CoV-2 pandemic. A country that was proactive in implementing measures such as social distancing, quarantine, orders to stay at home, testing symptomatic and asymptomatic loads and hygienic measures to limit the impacts of SARS-Cov-2 (China) and that today is clearly in the decay phase with the number of cases tending to zero and is no longer in a pandemic situation (efficient). Moderately proactive countries, ie, implemented measures only when the spread of SARS-Cov-2 was already reaching the country (France, Germany, United Kingdom, Spain, Sweden, Italy, Ireland, USA, Austria, and Canada) (moderately efficient) and the reactive countries, which took a long time to implement the measures and/or the infection came later and as a result are not managing to reduce the number of daily cases of SARS-CoV-2 (Russia, Iran, Brazil, and India) (inefficient) and are the new epicenters of the SARS-COV-2 pandemic. Besides, we applied the Bandt Pompe permutation entropy $(H)$ and the Fisher Information $(F)$ to obtain the rank of the most efficient countries to the fight against the SARS-CoV-2. To the best of our knowledge, no researches have been ranking the most proactive countries in the fight against the SARS-CoV-2 dissemination. We truly believe that the empirical results showed in this research draws new perspectives that can collaborate in the formulation of more efficient healthy public policies to combat SARS-CoV-2 spread.

\section{Introduction}

Severe acute respiratory syndrom coronavirus 2 (SARS-CoV-2) is a zoonotic emerging RNA single-strand enveloped virus that infects human and have caused pandemic outbreak disease since the last of $2019^{1}$. SARS-CoV-2 belongs to the Coronaviridae Family, which contains six other human coronavirus. Four of them (OC43, 229E, NL63, and HKU1) cause mild illness similar to the common cold and gastrointestinal tract infection. The other two, severe acute respiratory syndrome coronavirus (SARS-CoV) and Middle East respiratory syndrome coronavirus (MERS-CoV), have caused epidemic outbreak in the past, leading to high pathogenicity and mortality in humans ${ }^{2}$. The coronavirus disease-2019 (COVID-19), caused by SARS-CoV-2 begins with flu-like symptoms, some patients also have runny nose, nausea, vomiting, and diarrhea, and complications can lead to severe infections, such as pneumonia (infection of the lungs), kidney failure, and death ${ }^{3}$.

SARS-CoV-2 was first described in Wuhan city, Hubei province, China, in December 2019 and quickly spread to other provinces of China and other countries via international travelers. Since then, SARS-CoV-2 has been considered a Public Health Emergency of International Concern by World Health Organization (WHO $)^{4}$. About only a month later its discovery in China, the SARS-CoV-2 had already spread to several countries in Asia and Europe and in the US ${ }^{1}$. As of 16 March 2020, more than 150 countries and territories have been affected, with major outbreaks in central China, South Korea, Italy, Iran, France, and Germany ${ }^{5}$.

At that time, the number of confirmed cases and deaths in other countries than China have dramatically increased and the Europe was considered by WHO the new center of the pandemic. In April 2020, most of COVID-19 cases occurred in the $\mathrm{USA}^{1,6}$, and after that, other coutries have been highlighted as the source of new number cases, such as Brazil and Russia. 
A variety of preventive strategies have been put in place by different governments across the world. These strategies include testing symptomatic and asymptomatic carries, social distancing, quarantine, stay-at-home orders, and hygienic measures ${ }^{1,7}$. These strategies had different effects in diverse countries according to the time they were applied and other factors.

Thus, the chief goal of this research is to investigate the temporal evolution for SARS-CoV-2 pandemic consider the daily incidence number of COVID-19 cases for 15 countries based on the Shannon-Fisher causality plane (SFCP) ${ }^{8,9}$. Thus, we applied the Bandt \& Pompe permutation entropy ${ }^{10}$ and Fisher Information measure ${ }^{11,12}$ which allowed us to map and rank the countries most efficient in controlling the SARS-CoV-2 pandemic. The SFCP is a powerful tool of Information Theory to investigate the global and local characteristics of the Bandt Pompe's probability density function (PDF) related to the dynamics of SARS-CoV-2 dissemination.

The highlights of this research for the literature are: First, it analysis the complex dynamics of SARS-CoV-2 pandemic through the estimation of the Bandt \& Pompe permutation entropy combined with Fisher Information measure. Second, it builds up the SFCP for SARS-CoV-2, which allowed us to map the virus dynamic behaviour and their respective locations along the SFCP for each country. Third, it shows the ranking of the countries most efficiently in controlling the SARS-CoV-2 pandemic taking into account the complexity hierarchy.

\section{Results}

The insights promoted by our fitting procedure are based on data comprising of the number of daily incidences related of COVID-19 cases in 15 countries (Methods section for more details). For each time series, we investigate the phenomenology inherent to the temporal evolution of SARS-CoV-2 pandemic. These types of time series are widely known to be non-stationary. Despite that, the time series of the daily number of COVID-19 cases can be used to characterize the fractional Brownian motion, also a non-stationary process ${ }^{13-15}$.

Given this, taking into account the permutation entropy as a way to discriminate time series, the previous empirical results showed that better results ${ }^{16,17}$ are provided by original series, in this case is the daily incidence of SARS-CoV-2 infection. For each country, the time series of temporal evolution of SARS-CoV-2 pandemic are shown in Fig. 1.

The temporal evolution of the number of daily incidences related to COVID-19 depicts three distinct phases: an exponential increase phase, a plateau phase, and a decrease phase. In view of this, it seems that China, France, and Germany are in the decrease phase, while Brazil and India are in the exponential increase. In turn, Iran deserves attention once it peaked at April followed by a decay phase and then a new increase since May. While, the US presented a critical exponential increase phase, but it seems systematically decrease.

The SARS-CoV-2 pandemic generated strong negative effects into the investigated countries in this research, causing abrupt changes in the worldwide status quo of the people lives ${ }^{7}$. For each country, we perform the Boxplot ${ }^{18}$ to verify the anomalous values (outliers). Fig. 2 presents the Boxplot.

The analysis of Fig. 2 reveals the number of outliers for each country. It is an indication that extreme events in the time series of the daily incidence of COVID-19 are more recurrent for Austria, Brazil, China, France, Germany, India, Ireland, and Spain. Extreme events can be understood as statistically improbable occurrences. Even with a high probability of not occurring, these extreme events are observed in financial, social, and natural systems, bringing hard consequences ${ }^{19}$.

In this way, we also applied the univariate quantile-quantile (Q-Q) plot $^{20}$, which is a powerful tool used to examine the distributional similarities and differences between two independent samples ${ }^{20-22}$. Fig. 3 depicts the Q-Q plot.

The Q-Q plot is a non-parametric approach more robust than the classical histogram to study the statistical properties inherent to measures of central tendency, dispersion and skewness. For all countries investigated in this analysis, there is a standard statistical behavior in relation to the daily observations of COVID-19 cases. Especially at the ends of the Q-Q plot, the daily incidences follow a strong non-linear pattern, revealing that the data are not distributed by a Gaussian $(X \sim N(0,1))$.

Based on the Information Theory quantifiers, Permutation entropy $(H)$, Statistical complexity $(C)$, and Fisher Information $(F)$, all measures evaluated by Bandt \& Pompe method, it is possible to define three causality information planes ${ }^{23}$. Here, we use the causality Shannon-Fisher plane $(H x F)$ which performs an analysis based on the global and local characteristics of the Bandt Pompe's probability density function (PDF). We emphasize that there are some peculiars characteristics between the causality Shannon-Complexity plane $(H x C)$ and Shannon-Fisher causality plane $(H X F)$. The focus of $(H x C)$ comprises the global characteristics of the associated time series Bandt Pompe's probability density function (PDF). Thus, the range variation of $(H x C)$ includes $[0,1] x\left[C_{\min }, C_{\max }\right]^{24}$.

The Shannon-Fisher causality plane considers the global and local characteristics of the Bandt Pompe's PDF. The range is $[0,1] x[0,1]]$; no limit curves have been shown to exist so far ${ }^{25}$. This approach has already been used successfully to distinguish noise from chaos ${ }^{26}$, to investigate the characterization of motor, imagery movements in electroencephalogram ${ }^{27}$, to study the physiology of cerebral cortex ${ }^{23}$, to analyze the complex dynamics relative of observed and simulated ecosystem gross primary productivity ${ }^{25}$ and in the info-quantifiers to the logistic map ${ }^{9}$. The Shannon-Fisher causality plane are show in Fig. 4. 


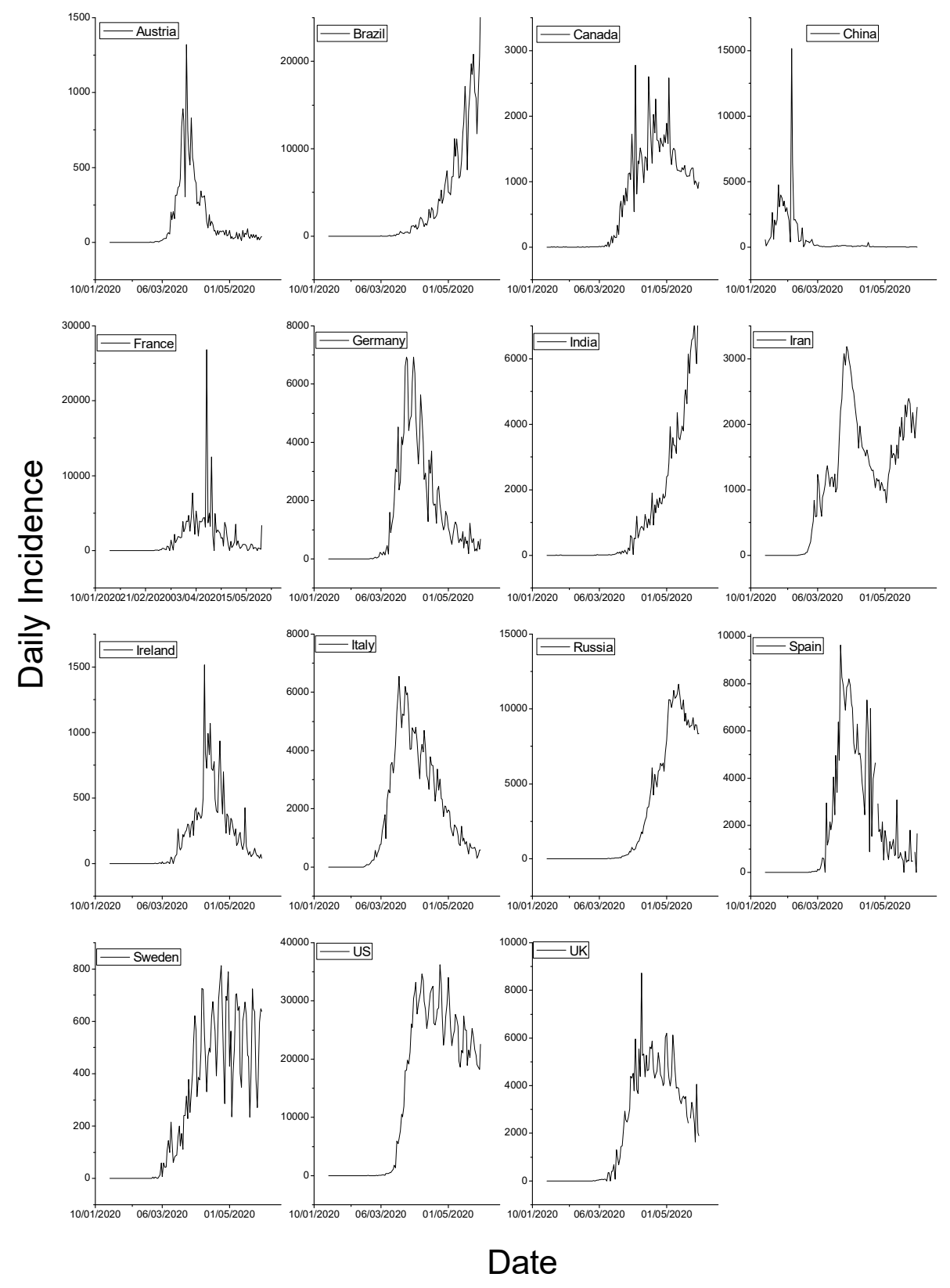

Figure 1. For each country, the temporal evolution of the number of daily incidence related to COVID-19 day from January 22, 2020 until May 282020 with 128 observations. These time series present peculiar characteristics such as non-linear dynamics, noisy and chaotic characteristics. 
China is the only country that is located within the ideal position zone to a stochastic process related to a random walk. It reveals that China is no longer in a pandemic situation and presents no community contagion. France is the country that is closest to entering this ideal position zone followed by Germany and the UK and will soon no longer be in a pandemic situation and is without presenting community contagion.

The other countries are far from this ideal position zone to a stochastic process related to a random walk. However, with the exception of Sweden, Brazil, India, Iran, and Russia the other countries are already in the decay phase. Sweden has a behavioral anomaly in terms of the spread of SARS-CoV-2. Apparently, it is the only one on the plateau phase. Brazil, India, Iran, and Russia are in the exponential growth phase related to the daily number of COVID-19 cases.

The SFCP method measures the magnitude of the total impact of the SARS-CoV-2 pandemic for the entire period of analysis. Thus, a temporal analysis of the number of COVID-19 cases is not carried out, but a general picture of the pandemic situation for the countries investigated. In this sense, what is being compared is the general picture of 15 countries, which allows us to verify which ones were most impacted considering the number of daily incidences SARS-CoV-2 infection.

The temporal evolution of the number of daily COVID-19 cases tends to have their starting positions close to the lower-right region at the Shannon-Fisher causality plane. The countries located in this region or in its surroundings are characterized by high entropy and low degree of reliability in relation to the information extracted from the analyzed data set, so their behavior is closer to a random walk. These countries have adopted more efficient measures in controlling the SARS-CoV-2 pandemic. These measures are directly associated with the fulfillment of the determinations of the World Health Organization (WHO) and with a greater political and social engagement we can highlights social distancing, quarantine, stay-at-home orders, testing symptomatic and asymptomatic loads and hygienic measures to control the SARS-CoV-2 pandemic.

While the countries located in the SFCP intermediate region or in its surroundings are characterized by a less entropic degree and a high degree of reliability in relation to the information extracted from the analyzed data set. These countries took a long time to adopt the measures recommended by the WHO and the political and social engagement was delayed as a result of that they still have a moderate risk of spreading by SARS-CoV-2.

The countries located to the top-right region at SFCP in its surroundings are characterized by lower entropy and higher degree of reliability in relation to the information extracted from the analyzed data set. These countries took even longer to adopt the measures recommended by the WHO and have a low level of political and social engagement in complying with these measures, thus presenting a high risk in relation to contagion by SARS-CoV-2. In addition, the situation related to this health crisis is enhanced by the collapse of the health system, poverty and a few years of educational studies.

The permutation entropy $H$ and Fisher information $F$ are used to quantify the degree of efficiency in combating the impacts of the spread of SARS-CoV-2 considering the time series of daily incidences of COVID-19 cases. For time series of the daily incidence of SARS-CoV-2, the time evolution is characterized by a temporal pattern derives in deviations from the ideal position to a stochastic process inherent a random walk. The higher distance to this random ideal position reflects a higher level of efficiency in the controlling the SARS-CoV-2 dissemination. Table 1 presents a rank of successful countries in controlling the SARS-CoV-2 pandemic and these impacts based on the complexity hierarchy.

Table 1. Rank of the countries based on the values of permutation entropy $H$, Fisher Information $F$ and distance from vertex $(1,0)$ considering $d=4$.

\begin{tabular}{rlrrr}
\hline Ranking & Country & \multicolumn{1}{c}{ Entropy } & Complexity & Dist to $(1,0)$ \\
\hline 1 & China & 0.96813 & 0.06177 & 0.069501 \\
2 & France & 0.92774 & 0.08716 & 0.113214 \\
3 & Germany & 0.9131 & 0.07268 & 0.11329 \\
4 & UK & 0.90078 & 0.09164 & 0.13506 \\
5 & Spain & 0.86532 & 0.10133 & 0.168544 \\
6 & Sweden & 0.85259 & 0.08509 & 0.170207 \\
7 & Italy & 0.86259 & 0.10349 & 0.172022 \\
8 & Ireland & 0.842635 & 0.11311 & 0.193798 \\
9 & US & 0.85919 & 0.14364 & 0.20115 \\
10 & Austria & 0.839348 & 0.16896 & 0.233142 \\
11 & Canada & 0.86678 & 0.19313 & 0.234619 \\
12 & Russia & 0.81272 & 0.14339 & 0.23587 \\
13 & Iran & 0.80657 & 0.14419 & 0.24126 \\
14 & Brazil & 0.81614 & 0.16898 & 0.249716 \\
15 & India & 0.83611 & 0.18986 & 0.25081 \\
\hline
\end{tabular}


For each country, the higher distance to point (1.0) reflects a higher level of efficiency in controlling the SARS-CoV-2 pandemic. In this sense, the results presents that China, France, Germany and U.K. are near to the lower boundary of the Shannon-Fisher causality plane. Because of this, they can be considered more efficient countries ${ }^{16,28-30}$. Moreover, the Euclidian distance presents a relevant discrepancy in the distance between China and France. It reveals that China is less complex per entropy value than France.

Otherwise, the lower distance from point (1.0) reflects less level of efficiency in the combat against to the SARS-CoV-2. Our results show that India, Brazil, Russia and Iran are lying significantly farther from the right corner leads behavioral dynamic related to SARS-CoV-2 far from a random walk. Thus, these countries are less efficient ${ }^{16}$ and show long-term correlated. Moreover, these countries are characterized by lower entropy and a lower degree of randomness. Given this, these countries are the most inefficient in the combat against to the SARS-CoV-2.

\section{Discussion}

Based on the empirical results presents in Fig. 1, Fig. 2, Fig. 3, Fig. 4 and Table 1 we perform individual analyzes to understand the complex phenomenology inherent of the number of daily incidence of COVID-19 cases. Our results show that we can cluster the 15 countries investigated into 3 different groups related to the SARS-CoV-2 pandemic, the efficient countries, the moderately efficient countries and the inefficient countries.

China is located in a region of the highest entropy and lowest Fisher information. These results may be a consequence of a quick and proactive stance in controlling the SARS-CoV-2 spread since had emerged the epidemic outbreak disease ${ }^{31}$, although it had a high peak in the daily incidence of COVID-19 cases in the beginning of the pandemic. China has been preparing to contain future pandemics by applying lessons learnt from SARS ever since $2003^{32}$. Within a matter of weeks, China implemented all the tools ranging from case detection with immediate isolation, and contact tracing with quarantining and medical observation of all contacts ${ }^{33}$.

Although Europe does not have the same preparation than China, Germany, France and U.K. are the countries closest to reaching this stage that today is China, a country that has a lower daily incidence of SARS-CoV-2 infection rate tending to zero. These countries clearly have a drastic reduction in COVID-19 cases in the last days studied. However, they did not invested in the strategy of mass testing, contact tracing, and physical distancing in the early stage of SARS-CoV-2 spread which led them to failures in inicial stage containment of COVID-19 pandemic ${ }^{34,35}$ But, we believe that public health policies implemented together with some factors such as population adherence to social isolation measure, genetic and age composition ${ }^{36}$ and high human development index (HDI) may have helped in decreasing the impact of SARS-CoV-2 in these countries.

Spain, Italy, Ireland, United States, Austria and Canada, already presenting a curve of cases confirmed by the decaying SARS-CoV-2, but still indicating a moderate risk of having new outbreaks if sanitary measures are not followed and there is early relaxation. Sweden has adopted more relaxed strategy to control the SARS-CoV-2 pandemic with no massive testing on suspected individuals and no strict lockdown in her most affected regions and, probably, because of this, we can observe a difference in a pattern of COVID-19 cases.

In the top-right region investigated by the Shannon-Fisher causality plane are Russia, Iran, Brazil and India. These countries are presenting high daily cases of SARS-CoV-2, their curves are growing and, therefore, their position on the Shannon-Fisher causality plane, present greater distance to the point of efficiency. Brazil and Iran had confirmed cases later than the others countries already mentioned, which contributed to being in the pandemic control stage presented. Among the factors that may be influencing Russia in its low efficiency and the response to COVID-19, the lack of clear political leadership has been pointed out, just like in Brazil ${ }^{37}$.

Brazil and India are two very populous countries that are undergoing economic development. Facing an outbreak of COVID-19 with these characteristics is quite challenging. Specifically in the case of Brazil, where we are familiar with the situation, several factors such as a permissive culture, political polarization, ${ }^{38}$ high social inequality, poor sanitation in many regions, public transportation often crowded in large cities, among other characteristics may be contributing enormously to the low efficiency in controlling the pandemic.

In conclusion, our results reveal different behavioral dynamics of the SARS-CoV-2 epidemic in the diverse countries evaluated. It is important to have instruments in order to analyze the country's efficiency in controlling the pandemic so that it is possible to know the strategies that were used by the most successful countries. Thus, a more effective response can be taken in the face of a new SARS-CoV-2 outbreak, or by countries that are still experiencing the first outbreak. However, it is worth noting that each country has its own intrinsic economic, social, behavioral, genetic, and age characteristics, among others, that will influence the final result, bringing about a more or less effective control of SARS-CoV-2 outbreaks. 


\section{Methods}

This section has been segregated into 2 distinct subsections in which we present the theoretical framework of the methods used in this research. The Bandt \& Pompe method (BPM) and the Shannon-Fisher Causality plane (SFCP). Fig. 5 depicts the flowchart related to the theoretical framework used in this research.

\section{Data}

The main database used in our analysis is the time series of daily incidence related to SARVS-COV-2 taking into account 19 countries. For each country, the periods cover 122 day from January 22, 2020 until May 282020 with 128 observations. The data were obtained via public service on the internet at COVID-19 Dashboard by the Center for Systems Science and Engineering (CSSE) at Johns Hopkins University,https: / / github. com/CSSEGISandData/COVID-19. According to the terms of use, the data is public and can be used for public health, educational, and academic research purpose. A list of countries considered in this research with country name and the respectively geographical coordinates and Human Development Index (IDH) is presented in Table 2.

Table 2. For each country, details inherent of they respective geographical coordinates and IDH. IDH were obtained in its annual Human Development Report related to 2019.

\begin{tabular}{ccccl}
\hline $\mathrm{i}$ & Country & Latitude & Longitude & IDH \\
\hline 1 & Austria & 47.5162 & 14.5501 & 0.914 \\
2 & Brazil & -14.235 & -51.9253 & 0.761 \\
3 & Canada & 56.1303 & -106.3467 & 0.922 \\
4 & China & 39.9138 & 116.3636 & 0.758 \\
5 & France & 46.2276 & 2.2137 & 0.891 \\
6 & Germany & 51.0 & 9.0 & 0.939 \\
7 & India & 21.0 & 78.0 & 0.647 \\
8 & Iran & 32.0 & 53.0 & 0.797 \\
9 & Ireland & 53.1424 & -7.6921 & 0.942 \\
10 & Italy & 43.0 & 12.0 & 0.883 \\
11 & Russia & 60.0 & 90.0 & 0.824 \\
12 & Spain & 40.0 & -4.0 & 0.893 \\
13 & Sweden & 63.0 & 16.0 & 0.937 \\
14 & UK & 55.378 & -3.4359 & 0.92 \\
15 & US & 37.0902 & -95.7129 & 0.92 \\
\hline
\end{tabular}

Specifically, the HDI is an index that considers in its calculation 3 variables, life expectancy at birth, expected years of schooling for children and mean years of schooling for adults, and per capita income.

In this research, we taking into account countries that present very high human development (Austria, Canada, France, Germany, Ireland, Italy, Russia, Spain, Sweden, U.S. and U.K.), high human development (Brazil, China and Iran) and medium human development (India).

For each country, regardless of the value related to its HDI, it is notable that SARVS-CoV-2 has dramatically altered social, economic structures and the health system. Enforcing the isolation of people on a global scale.

\section{Permatutation entropy}

The Permutation Entropy (PE) approach was proposed by Bandt \& Pompe $^{10}$ as the Shannon's entropy ${ }^{39}$ to quantify the probability distribution of ordinal patterns which are constructed through the symbolization technique that considers time causality by comparing neighboring values of the time series under analysis ${ }^{40,41}$.

The permutation entropy is a measure for information content in time series ${ }^{42}$ which is apply to evaluate uncertainty, disorder, state-space volume, and lack of information ${ }^{43}$ inherent in a process as displayed by discrete measurements of a parameter of the system ${ }^{44}$.

Thus, more predictable signals (which show a tendency to repeat only a few ordinal patterns) have lower permutation entropy than less predictable signals (which tend to exhibit all possible ordinal patterns).

This method taking into account a symbolic sequence of ordinal patterns of segments (words) of a given size (embedding dimension) denoted by $d^{16,40}$. Then, for each ordinal pattern the respective instances are counted in order to construct an 
ordinal pattern probability distribution ${ }^{17}$. More specifically, these ordinal patterns related to the quantifying of Bandt $\&$ Pompe probability distribution are calculated by observing the local ordering of consecutive values within each word.

In view of this, the Bandt \& Pompe method (BPM) can be described as follows. For a given time series $x_{t}, t=1, \ldots, T$ initially $T-(d-1)$ overlapping segments $X_{t}=\left(x_{t}, x_{t+1}, \ldots, x_{t+d-1}\right)$ of length $d$ (incorporation dimension) are generated and within each segment the values are classified in ascending order, which provides the set of indices $r_{0}, r_{1}, \ldots, r_{d-1}$ such that $x_{t+r_{0}} \leq x_{t+r_{1}} \leq \ldots \leq x_{t+r_{d-1}}$. The corresponding sequences denoted by $\pi=r_{0}, r_{1}, \ldots, r_{d-1}$ can take on any of $d$ ! possible permutations of the set $\{0,1, \ldots, d-1\}$ and are symbolic representatives of the original segments. The permutation entropy of order $d \geq 2$ is now defined as a Shannon entropy of probability distribution $P(\pi)$, given by

$$
H(d)=-\sum_{\pi} p(\pi) \log p(\pi)
$$

where $\{\pi\}$ represents the summation over all the $d$ ! possible permutations of order $d$, and $p(\pi)$ denotes the relative frequency of occurrences of the permutation $\pi$. It follows $0 \leq H(d) \leq \log d$ ! t follows that $0 \leq H(d) \leq \log d$ ! where the lower limit is reached for a strictly increasing or decreasing series (only one permutation appears), and the upper limit for a completely random series where all $d$ ! Possible permutations appear with the same probability. The ideal $d$ strongly is related to the phenomenology of each event studied, but in order to promoted a goodness of fit statistics as a rule of thumb it is typically recommended ${ }^{40}$ to choose maximum $d$ such that $T>5 d$ !.

In addition, the application of BPM is recommended not only to time series of low dimensional dynamical systems, but also to any type of time series such as regular, chaotic, noisy or reality based $)^{29}$. Given this characteristic, the BPM has been successfully applied in distinct research areas such finance ${ }^{28,29,41}$, geophysics ${ }^{45}$, engineering ${ }^{46-48}$, physiology ${ }^{49-51}$, hydrology 52,53 and climatology 54

\section{Fisher information}

Fisher (1922) proposed a statistical measure of indeterminacy now called Fisher Information ${ }^{55}$. There are some possibilities for interpreting this statistical measure (i) as a measure of the ability to estimate a parameter, (ii) as the amount of information that can be extracted from a set of measures (the "quality" of the measurements) and (iii) as the disorder state measure of a system or phenomenon ${ }^{11,12,56}$ being its most important property is the so-called the Cramer-Rao Bound (CRB) for nonlinear parameter estimation ${ }^{9,57-60}$. The Cramer-Rao inequality ${ }^{26}$ is obeyed by all time series of quarterly GDP'S.

Specifically, Fisher information is a robust measure given by

$$
F[f]=\int \frac{|\vec{\nabla} f(x)|^{2}}{f(x)} d x
$$

which evaluate the gradient content of the distribution $f$ (continuous PDF), thus being quite sensitive even to tiny localized perturbations.

In view of this, it is essential to note that the gradient operator significantly influences the contribution of tiny local $f$-variations to the value of Fisher's information, so that the quantifier is called "local". Note that Shannon's entropy decreases with the skewed distribution, while Fisher's information increases in this case. Local sensitivity is useful in scenarios whose description requires an appeal to a notion of "order"8,9,26.

The concomitant problem regarding loss of information due to discretization has been thoroughly studied, for more details see ( ${ }^{61-63}$ and their references) and, in particular, it implies the loss of Fisher's shift invariance, which has no importance for our current purposes. For the calculation of the Fisher information measure (discrete PDF), we follow the proposal of Dehesa and collaborators ${ }^{64}$ based on the amplitude of the probability $f(x)=\psi(x)^{2}$ so,

$$
F(\psi)=4 \int\left\{\frac{\partial \psi}{\partial x}\right\} \partial x
$$

its discrete normalized version $(0 \leq F \leq 1)$ is defined by

$$
F[P]=\sum_{i=1}^{N-1}\left(\sqrt{P_{i+1}}-\sqrt{P_{i}}\right)^{2}
$$


In this paper the normalization constant $F_{0}$ reads

$$
F_{0}=\left\{\begin{array}{l}
1, \text { if } P_{i *} \text { for } i *=N \text { and } P_{i}=0 \forall i \neq i * \\
1 / 2 \text { otherwise. }
\end{array}\right.
$$

Based on the level of ordering of the investigated system, we can considering that it describe by a probability density function (PDF) given by $P_{o}=\left\{p_{k} \cong 1 ; p_{i} \cong 0 \forall i \neq k ; i=1, \ldots, N\right\}$ (with $N$, the number of states of he system) in consequence we have a Shannon entropy $S\left[P_{o}\right] \cong 0$ and a normalized Fisher's information measure $F\left[P_{o}\right] \cong F_{\max }=1$. Otherwise, if the investigated system is represented by a very disordered state, we can understand this particular state is described by a PDF given by the uniform distribution $P_{e}=\left\{p_{i}=\frac{1}{N} \forall i=1, \ldots, N\right\}$.

\section{Acknowledgement}

The authors are grateful to the Center for Systems Science and Engineering (CSSE) at Johns Hopkins University for making the data publicly available through COVID-19 dashboard and thus being used in this research.

\section{References}

1. Helmy, Y. A. et al. The covid-19 pandemic: a comprehensive review of taxonomy, genetics, epidemiology, diagnosis, treatment, and control. J. Clin. Medicine 9, 1225 (2020).

2. Wu, A. et al. Genome composition and divergence of the novel coronavirus (2019-ncov) originating in china. Cell host \& microbe (2020).

3. Huang, C. et al. Clinical features of patients infected with 2019 novel coronavirus in wuhan, china. The lancet $\mathbf{3 9 5}$, 497-506 (2020).

4. Raturi, M. \& Kusum, A. The active role of a blood center in outpacing the transfusion transmission of covid-19. Transfus. Clinique et Biol. 27, 96 (2020).

5. Organization, W. H. et al. Coronavirus disease (covid-2019) situation reports. 2020. Available on: https://www. WHO. Int/docs/default-source/coronaviruse/situationreports/20200221-sitrep-32-covid 19 (2020).

6. GHOSAL, S., Sinha, B., Sengupta, S. \& Majumder, M. Frequency of testing for covid 19 infection and the presence of higher number of available beds per country predict outcomes with the infection, not the gdp of the country-a descriptive statistical analysis. medRxiv (2020).

7. Jacobson, N. C. et al. Flattening the mental health curve: Covid-19 stay-at-home orders are associated with alterations in mental health search behavior in the united states. JMIR Mental Heal. 7, e19347 (2020).

8. Olivares, F., Plastino, A. \& Rosso, O. A. Ambiguities in bandt-pompe's methodology for local entropic quantifiers. Phys. A: Stat. Mech. its Appl. 391, 2518-2526 (2012).

9. Rosso, O. A., De Micco, L., Plastino, A. \& Larrondo, H. A. Info-quantifiers' map-characterization revisited. Phys. A: Stat. Mech. its Appl. 389, 4604-4612 (2010).

10. Bandt, C. \& Pompe, B. Permutation entropy: a natural complexity measure for time series. Phys. review letters $\mathbf{8 8 ,} 174102$ (2002).

11. Frieden, B. R. Science from fisher information. Sci. from Fish. Information, by B. Roy Frieden, pp. 502. ISBN 0521810795. Cambridge, UK: Camb. Univ. Press. July 2004. 502 (2004).

12. Mayer, A. L., Pawlowski, C. W. \& Cabezas, H. Fisher information and dynamic regime changes in ecological systems. Ecol. modelling 195, 72-82 (2006).

13. Rosso, O., Larrondo, H., Martin, M., Plastino, A. \& Fuentes, M. Distinguishing noise from chaos. Phys. review letters 99, 154102 (2007).

14. Bandt, C. \& Shiha, F. Order patterns in time series. J. Time Ser. Analysis 28, 646-665 (2007).

15. Zunino, L. et al. Permutation entropy of fractional brownian motion and fractional gaussian noise. Phys. Lett. A 372, 4768-4774 (2008).

16. Zunino, L., Zanin, M., Tabak, B. M., Pérez, D. G. \& Rosso, O. A. Complexity-entropy causality plane: A useful approach to quantify the stock market inefficiency. Phys. A: Stat. Mech. its Appl. 389, 1891-1901 (2010). 
17. Stosic, D., Stosic, D., Ludermir, T. B. \& Stosic, T. Exploring disorder and complexity in the cryptocurrency space. Phys. A: Stat. Mech. its Appl. 525, 548-556 (2019).

18. Tukey, J. W. Exploratory data analysis. (1977).

19. Anderson, S. C., Branch, T. A., Cooper, A. B. \& Dulvy, N. K. Black-swan events in animal populations. Proc. Natl. Acad. Sci. 114, 3252-3257 (2017).

20. Wilk, M. B. \& Gnanadesikan, R. Probability plotting methods for the analysis for the analysis of data. Biometrika 55, $1-17$ (1968).

21. Chambers, J., Cleveland, W., Kleiner, B. \& Tukey, P. Graphical methods for data analysis. wadsworth int'l. Group, Belmont, CA (1983).

22. Dhar, S. S., Chakraborty, B., Chaudhuri, P. et al. Comparison of multivariate distributions using quantile-quantile plots and related tests. Bernoulli 20, 1484-1506 (2014).

23. Montani, F., Deleglise, E. B. \& Rosso, O. A. Efficiency characterization of a large neuronal network: A causal information approach. Phys. A: Stat. Mech. its Appl. 401, 58-70 (2014).

24. Martin, M., Plastino, A. \& Rosso, O. Generalized statistical complexity measures: Geometrical and analytical properties. Phys. A: Stat. Mech. its Appl. 369, 439-462 (2006).

25. Sippel, S. et al. Diagnosing the dynamics of observed and simulated ecosystem gross primary productivity with time causal information theory quantifiers. PloS one 11 (2016).

26. Olivares, F., Plastino, A. \& Rosso, O. A. Contrasting chaos with noise via local versus global information quantifiers. Phys. Lett. A 376, 1577-1583 (2012).

27. Baravalle, R., Rosso, O. A. \& Montani, F. Causal shannon-fisher characterization of motor/imagery movements in eeg. Entropy 20, 660 (2018).

28. Zunino, L., Bariviera, A. F., Guercio, M. B., Martinez, L. B. \& Rosso, O. A. On the efficiency of sovereign bond markets. Phys. A: Stat. Mech. its Appl. 391, 4342-4349 (2012).

29. Bariviera, A. F., Guercio, M. B., Martinez, L. B. \& Rosso, O. A. A permutation information theory tour through different interest rate maturities: the libor case. Philos. Transactions Royal Soc. A: Math. Phys. Eng. Sci. 373, 20150119 (2015).

30. de Araujo, F. H. A., Bejan, L., Rosso, O. A. \& Stosic, T. Permutation entropy and statistical complexity analysis of brazilian agricultural commodities. Entropy 21, 1220 (2019).

31. Lau, H. et al. The positive impact of lockdown in wuhan on containing the covid-19 outbreak in china. J. travel medicine 27, taaa037 (2020).

32. ZHONG, N.-S. \& ZENG, G.-Q. Pandemic planning in china: applying lessons from severe acute respiratory syndrome. Respirology 13, S33-S35 (2008).

33. Wilder-Smith, A. \& Freedman, D. O. Isolation, quarantine, social distancing and community containment: pivotal role for old-style public health measures in the novel coronavirus (2019-ncov) outbreak. J. travel medicine 27, taaa020 (2020).

34. Khosrawipour, V. et al. Failure in initial stage containment of global covid-19 epicenters. J. Med. Virol. (2020).

35. Moatti, J.-P. The french response to covid-19: intrinsic difficulties at the interface of science, public health, and policy. The Lancet Public Heal. 5, e255 (2020).

36. Dowd, J. B. et al. Demographic science aids in understanding the spread and fatality rates of covid-19. Proc. Natl. Acad. Sci. 117, 9696-9698 (2020).

37. Lancet, T. Salient lessons from russias COVID-19 outbreak. The Lancet 395, 1739, DOI: 10.1016/s0140-6736(20)31280-0 (2020).

38. Van Bavel, J. J. et al. Using social and behavioural science to support covid-19 pandemic response. Nat. Hum. Behav. $1-12(2020)$.

39. Shannon, C. E. \& Weaver, W. The mathematical theory of communication, 117 pp. Urbana: Univ. Ill. Press. (1949).

40. Ribeiro, H. V., Zunino, L., Mendes, R. S. \& Lenzi, E. K. Complexity-entropy causality plane: A useful approach for distinguishing songs. Phys. A: Stat. Mech. its Appl. 391, 2421-2428 (2012).

41. Bariviera, A. F., Guercio, M. B., Martinez, L. B. \& Rosso, O. A. The (in) visible hand in the libor market: an information theory approach. The Eur. Phys. J. B 88, 208 (2015). 
42. Shannon, C. Tech. 27 (1948) 379; ce shannon, bell syst. Tech 27, 623 (1948).

43. Brissaud, J.-B. The meanings of entropy. Entropy 7, 68-96 (2005).

44. Weck, P. J., Schaffner, D. A., Brown, M. R. \& Wicks, R. T. Permutation entropy and statistical complexity analysis of turbulence in laboratory plasmas and the solar wind. Phys. Rev. E 91, 023101 (2015).

45. Ghil, M. et al. Advanced spectral methods for climatic time series. Rev. geophysics 40, 3-1 (2002).

46. Yan, R., Liu, Y. \& Gao, R. X. Permutation entropy: A nonlinear statistical measure for status characterization of rotary machines. Mech. Syst. Signal Process. 29, 474-484 (2012).

47. Wu, S.-D., Wu, P.-H., Wu, C.-W., Ding, J.-J. \& Wang, C.-C. Bearing fault diagnosis based on multiscale permutation entropy and support vector machine. Entropy 14, 1343-1356 (2012).

48. Zhang, X., Liang, Y., Zhou, J. et al. A novel bearing fault diagnosis model integrated permutation entropy, ensemble empirical mode decomposition and optimized svm. Measurement 69, 164-179 (2015).

49. Graff, G. et al. Ordinal pattern statistics for the assessment of heart rate variability. The Eur. Phys. J. Special Top. 222, 525-534 (2013).

50. Kalpakis, K. et al. Permutation entropy analysis of vital signs data for outcome prediction of patients with severe traumatic brain injury. Comput. biology medicine 56, 167-174 (2015).

51. Graff, B. et al. Entropy measures in the assessment of heart rate variability in patients with cardiodepressive vasovagal syncope. Entropy 17, 1007-1022 (2015).

52. Jovanovic, T., García, S., Gall, H. \& Mejía, A. Complexity as a streamflow metric of hydrologic alteration. Stoch. Environ. Res. Risk Assess. 31, 2107-2119 (2017).

53. Stosic, T., Stosic, B. \& Singh, V. P. Optimizing streamflow monitoring networks using joint permutation entropy. J. Hydrol. 552, 306-312 (2017).

54. Barreiro, M., Marti, A. C. \& Masoller, C. Inferring long memory processes in the climate network via ordinal pattern analysis. Chaos: An Interdiscip. J. Nonlinear Sci. 21, 013101 (2011).

55. Fisher, R. A. On the mathematical foundations of theoretical statistics. Philos. Transactions Royal Soc. London. Ser. A, Containing Pap. a Math. or Phys. Character 222, 309-368 (1922).

56. Frieden, B. R. Physics from fisher information: a unification (2000).

57. Blahut, R. E. \& Blahut, R. E. Principles and practice of information theory, vol. 1 (Addison-Wesley Reading, MA, 1987).

58. Zamir, R. A proof of the fisher information inequality via a data processing argument. IEEE Transactions on Inf. Theory 44, 1246-1250 (1998).

59. Pakrooh, P., Scharf, L. L., Pezeshki, A. \& Chi, Y. Analysis of fisher information and the cramér-rao bound for nonlinear parameter estimation after compressed sensing. In 2013 IEEE International Conference on Acoustics, Speech and Signal Processing, 6630-6634 (IEEE, 2013).

60. Dehesa, J., González-Férez, R. \& Sánchez-Moreno, P. The fisher-information-based uncertainty relation, cramer-rao inequality and kinetic energy for the d-dimensional central problem. J. Phys. A: Math. Theor. 40, 1845 (2007).

61. Zografos, K., Ferentinos, K. \& Papaioannou, T. Discrete approximations to the csiszár, renyi, and fisher measures of information. The Can. J. Stat. Revue Can. de Stat. 355-366 (1986).

62. Pardo, L., Morales, D., Ferentinos, K. \& Zografos, K. Discretization problems on generalized entropies and $r$-divergences. Kybernetika 30, 445-460 (1994).

63. Madiman, M., Johnson, O. \& Kontoyiannis, I. Fisher information, compound poisson approximation, and the poisson channel. In 2007 IEEE International Symposium on Information Theory, 976-980 (IEEE, 2007).

64. Sánchez-Moreno, P., Yánez, R. \& Dehesa, J. Discrete densities and fisher information. In Proceedings of the 14th International Conference on Difference Equations and Applications. Difference Equations and Applications. Istanbul, Turkey: Bahçesehir University Press, 291-298 (2009).

\section{Author contributions statement}

L.F. conceptualization, L.F. and F.A. data curation, L.F., F.A.and M.S. formal analysis, L.F., F.A., and M.S. investigation, L.F., and F.A. methodology, L.F. project administration, L.F., L.F. supervision, L.F., F.A., and M.S. validation, L.F., F.A., and M.S. visualization, L.F., F.A. and M.S. writing - original draft. All authors reviewed the manuscript. 


\section{Competing interests}

The authors declare that they have no known competing financial interests or personal relationships that could have appeared to influence the work reported in this paper. 


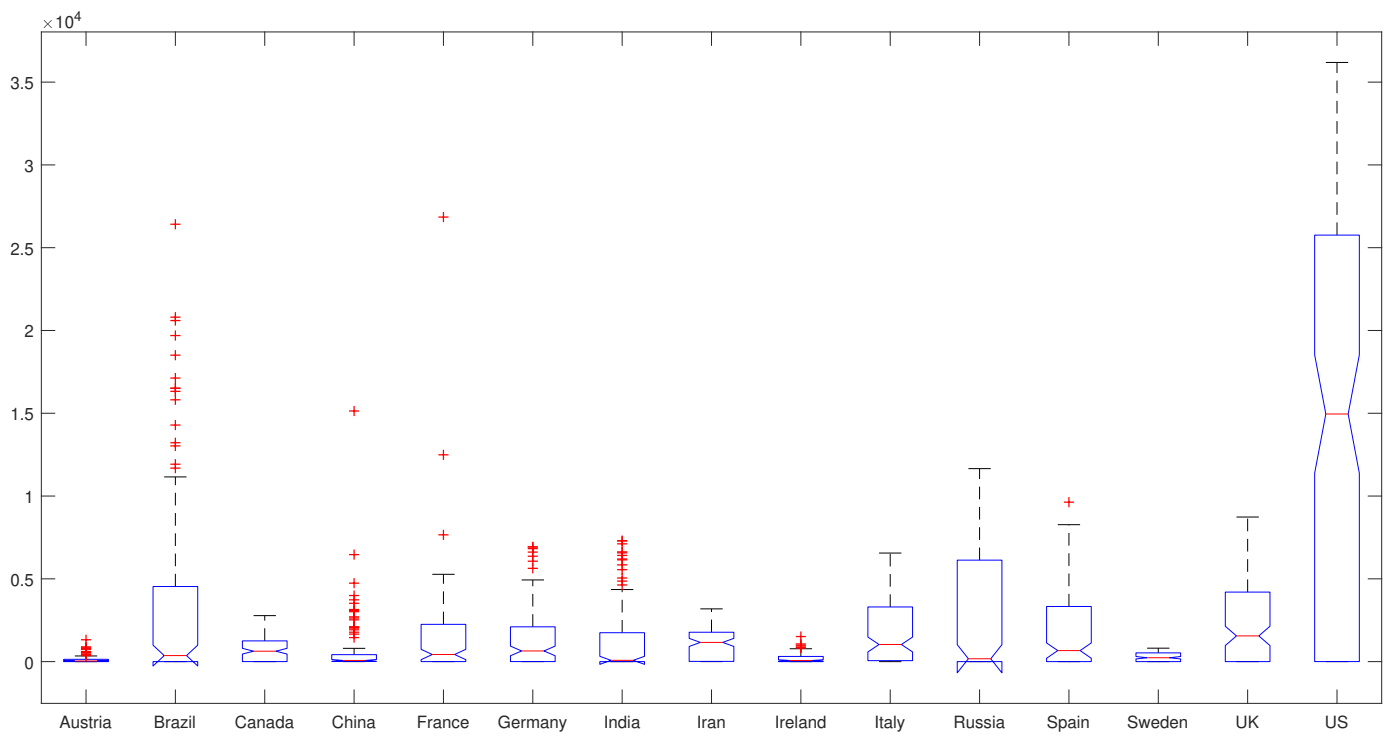

Figure 2. The median for the time series of the number of daily incidence related to SARS-CoV-2 showed variability for each country. Outliers are the points shown in red that are below the lower limit and above the upper limit. 

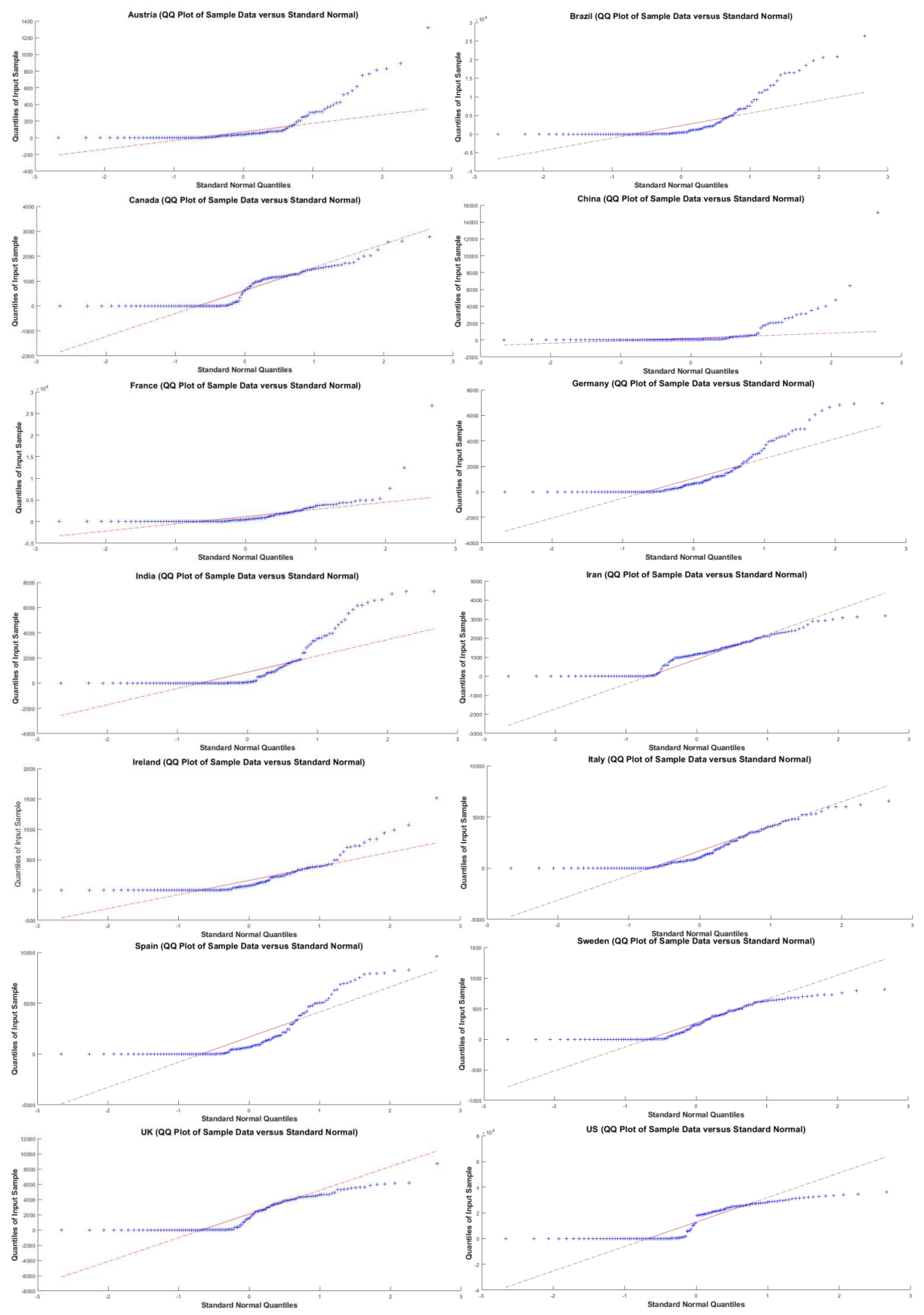

Figure 3. The plot produces an approximately straight line, suggesting that the number of daily incidences related to COVID-19 cases follows a non-gaussian distribution. 


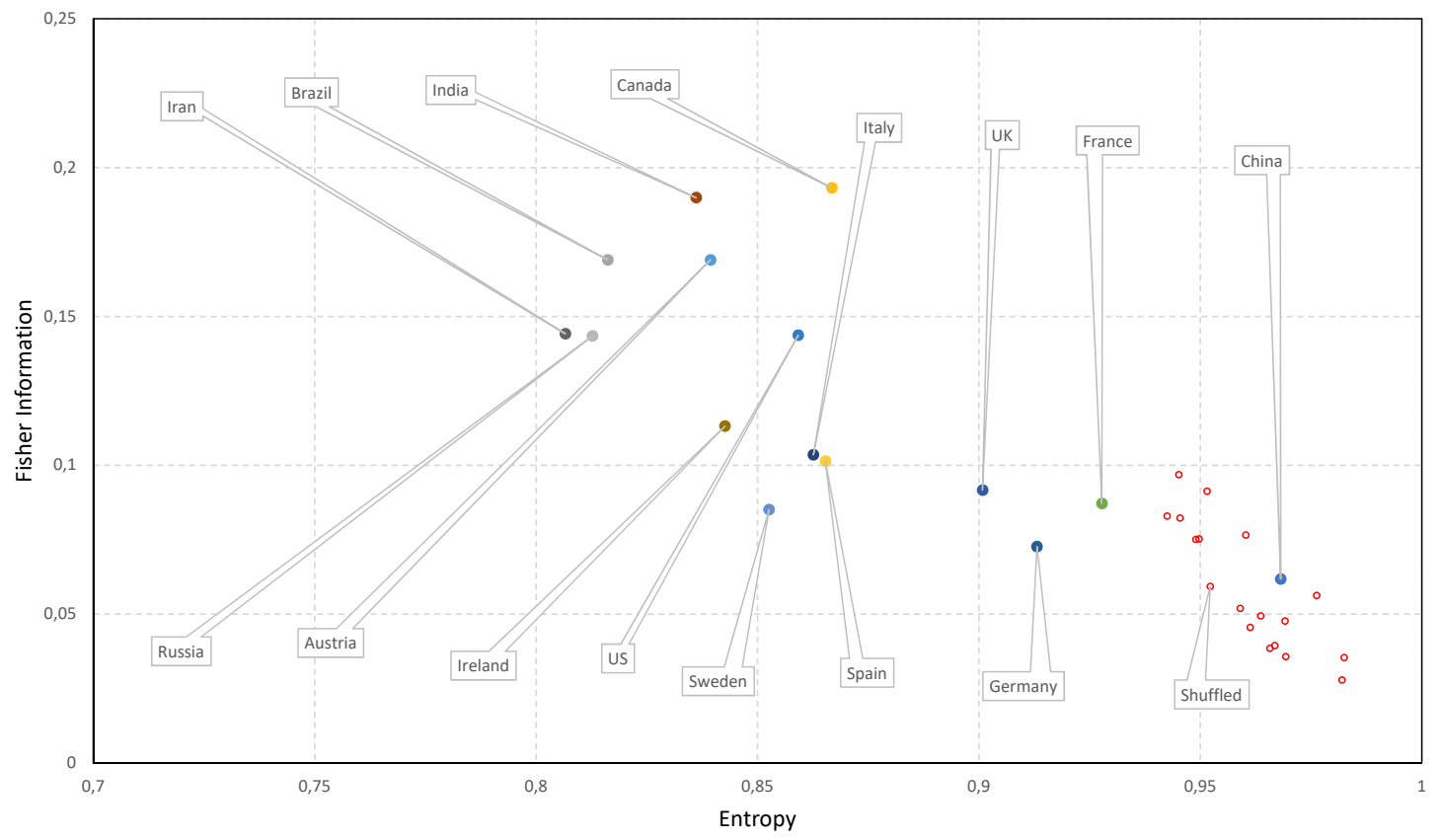

Figure 4. Illustration of the Shannon-Fisher causality plane $(H x F)$ is applied to study the temporal evolution of the daily number of COVID-19 cases. For each time series of the number of daily incidences related to COVID-19, the permutation entropy measures are calculated considering $d=4$ to satisfy the common condition $T>5 d$ !. 


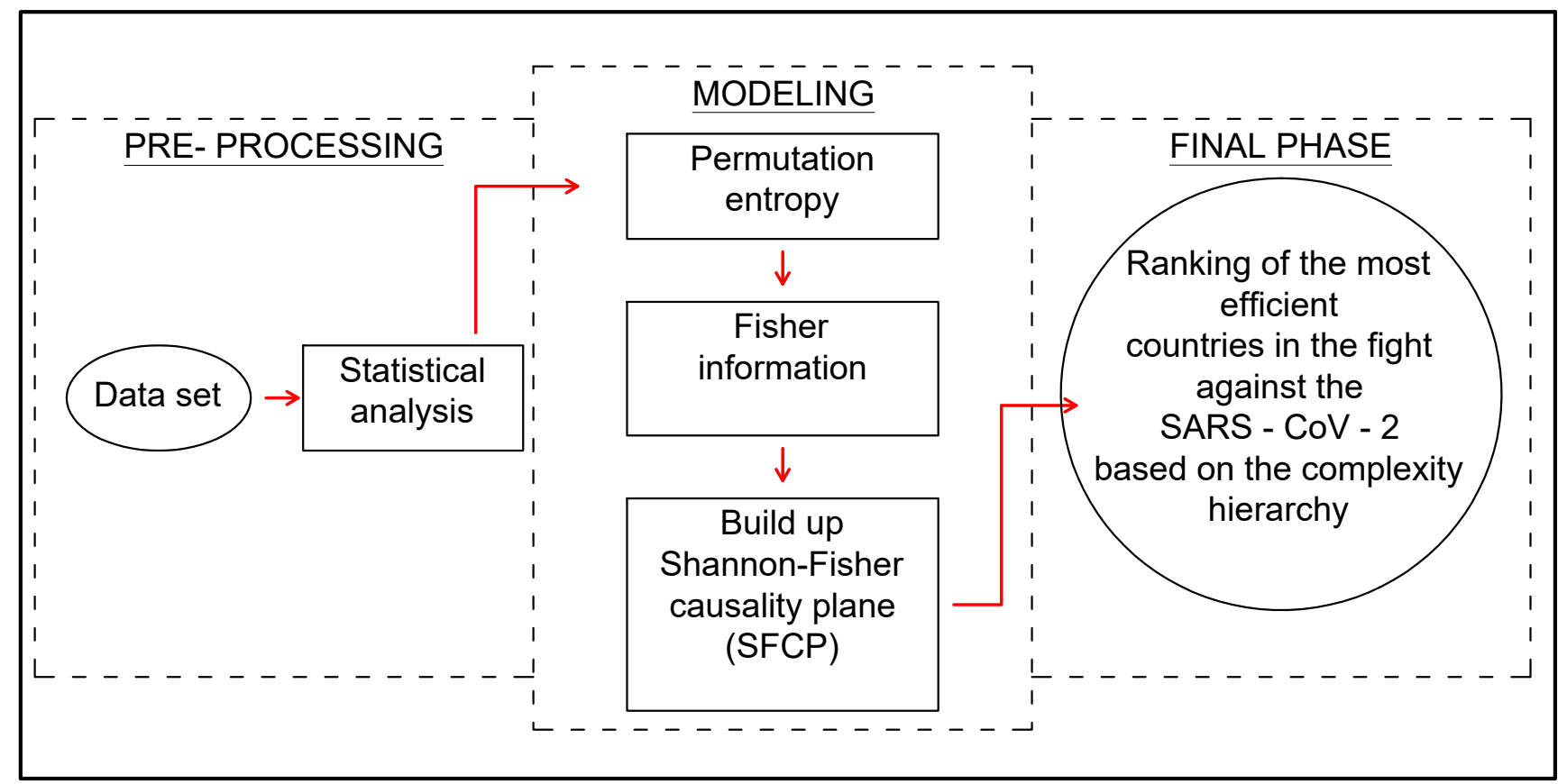

Figure 5. The flowchart of the theoretical framework of the procedures adopted in this research. Pre-processing corresponds to the analysis of data set and the application of statistical descriptive techniques. Modeling reflects the methods highlighted here and the final phase show the hierarchical complexity based on the Bandt \& Pompe permutation entropy $H$ and Fisher Information $F$. 


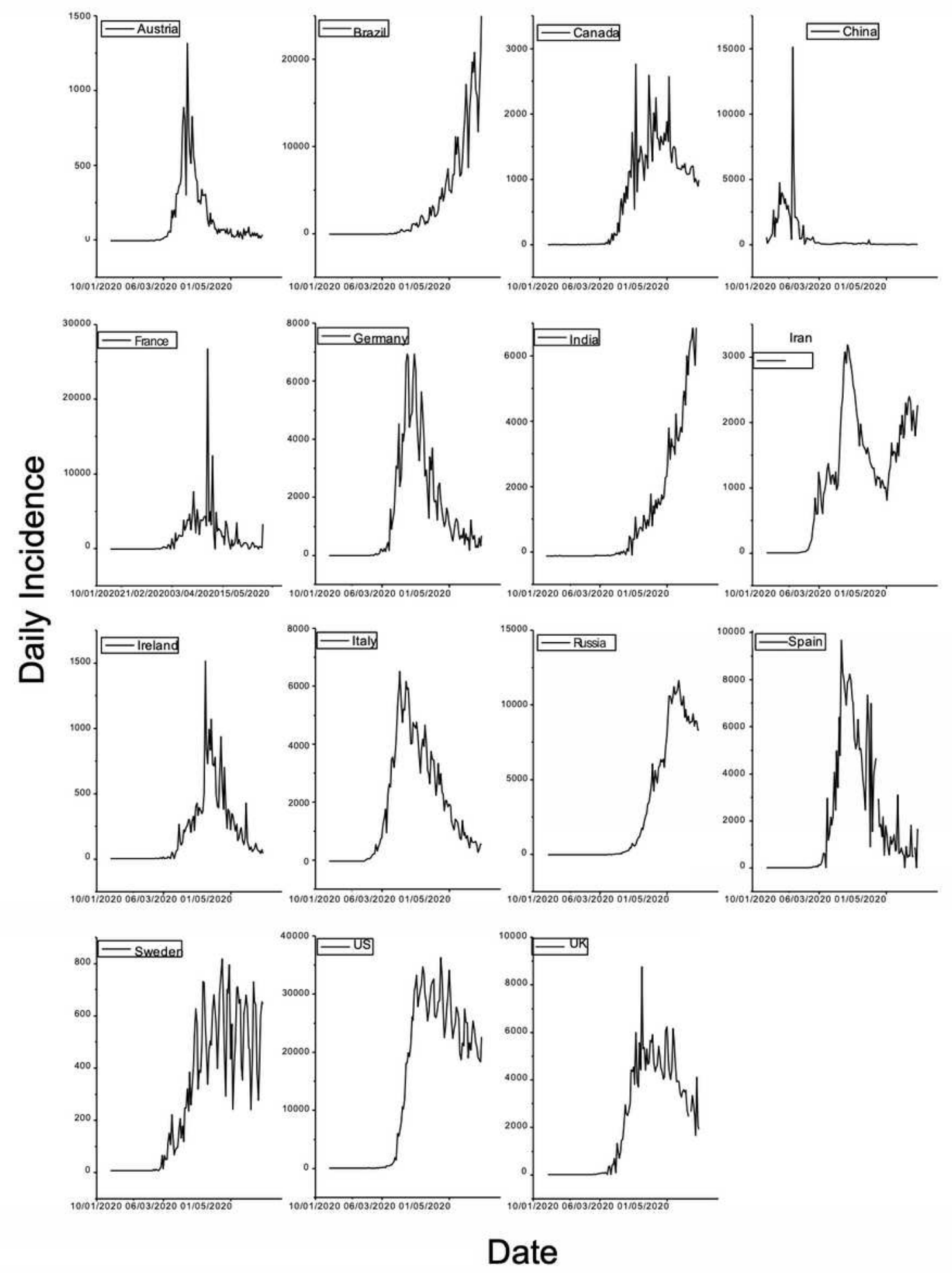

Figure 1

For each country, the temporal evolution of the number of daily incidence related to COVID-19 day from January 22, 2020 until May 282020 with 128 observations. These time series present peculiar characteristics such as non-linear dynamics, noisy and chaotic characteristics. 


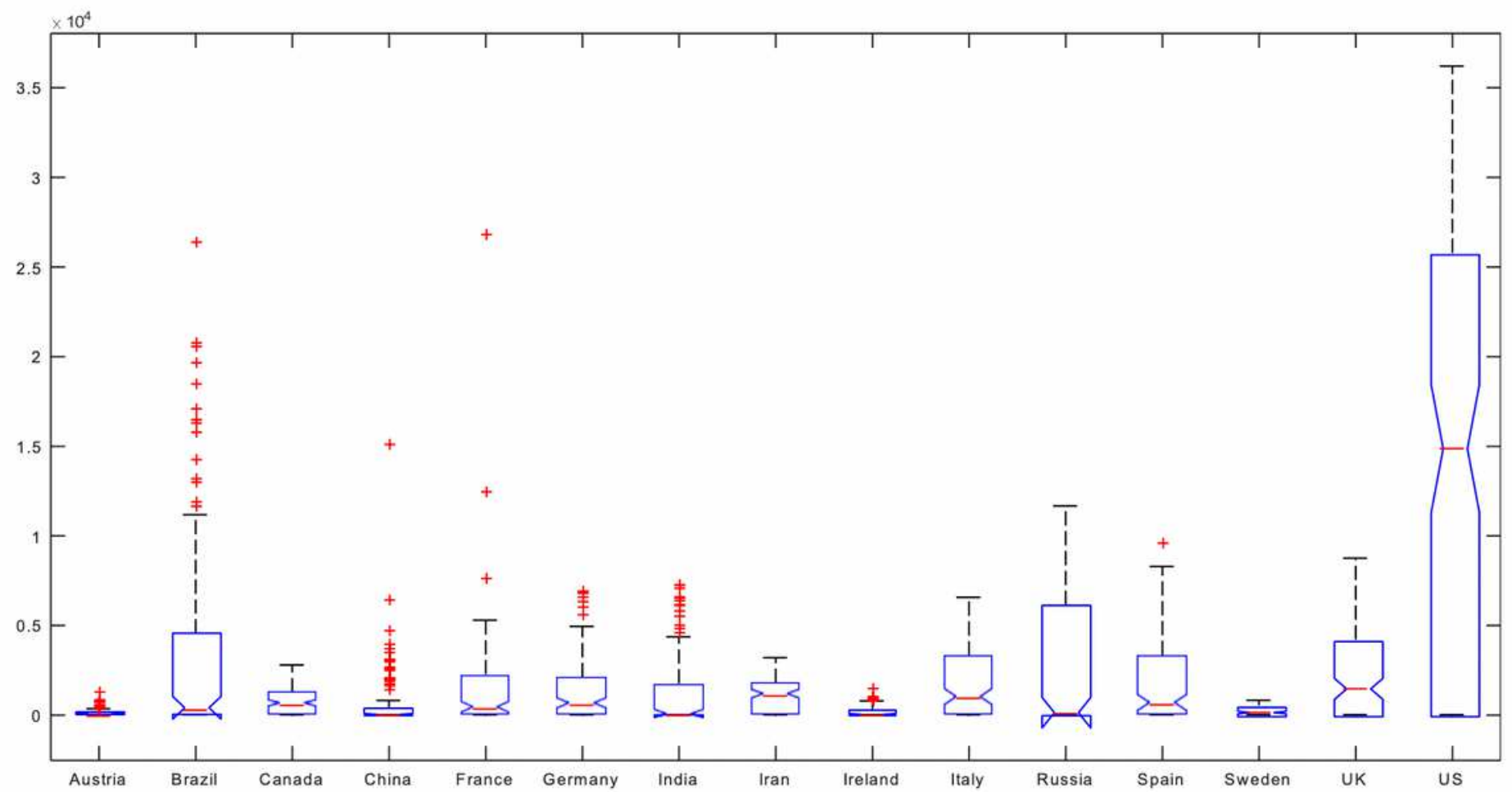

\section{Figure 2}

The median for the time series of the number of daily incidence related to SARS-CoV-2 showed variability for each country. Outliers are the points shown in red that are below the lower limit and above the upper limit. 


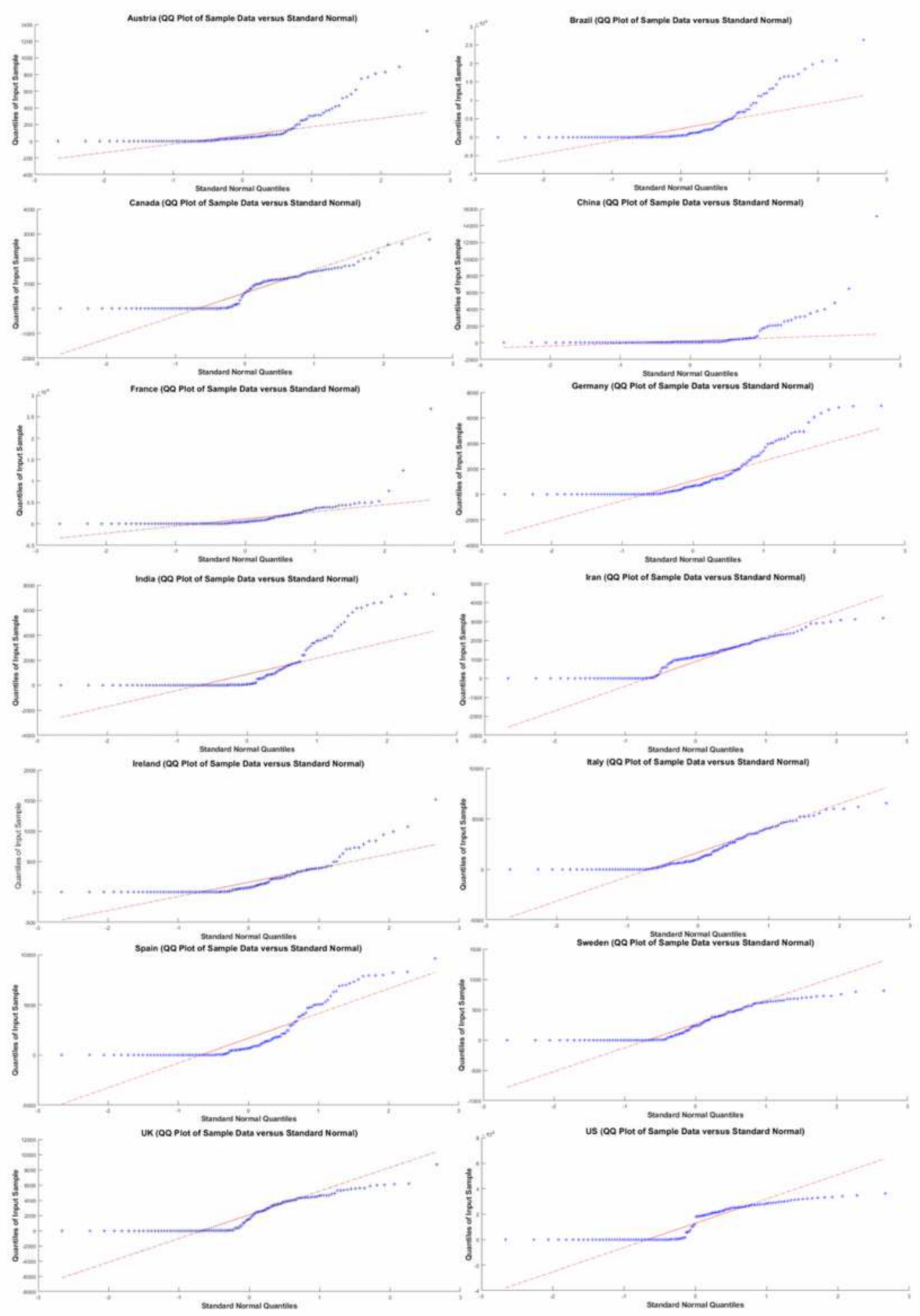

\section{Figure 3}

The plot produces an approximately straight line, suggesting that the number of daily incidences related to COVID-19 cases follows a non-gaussian distribution. 


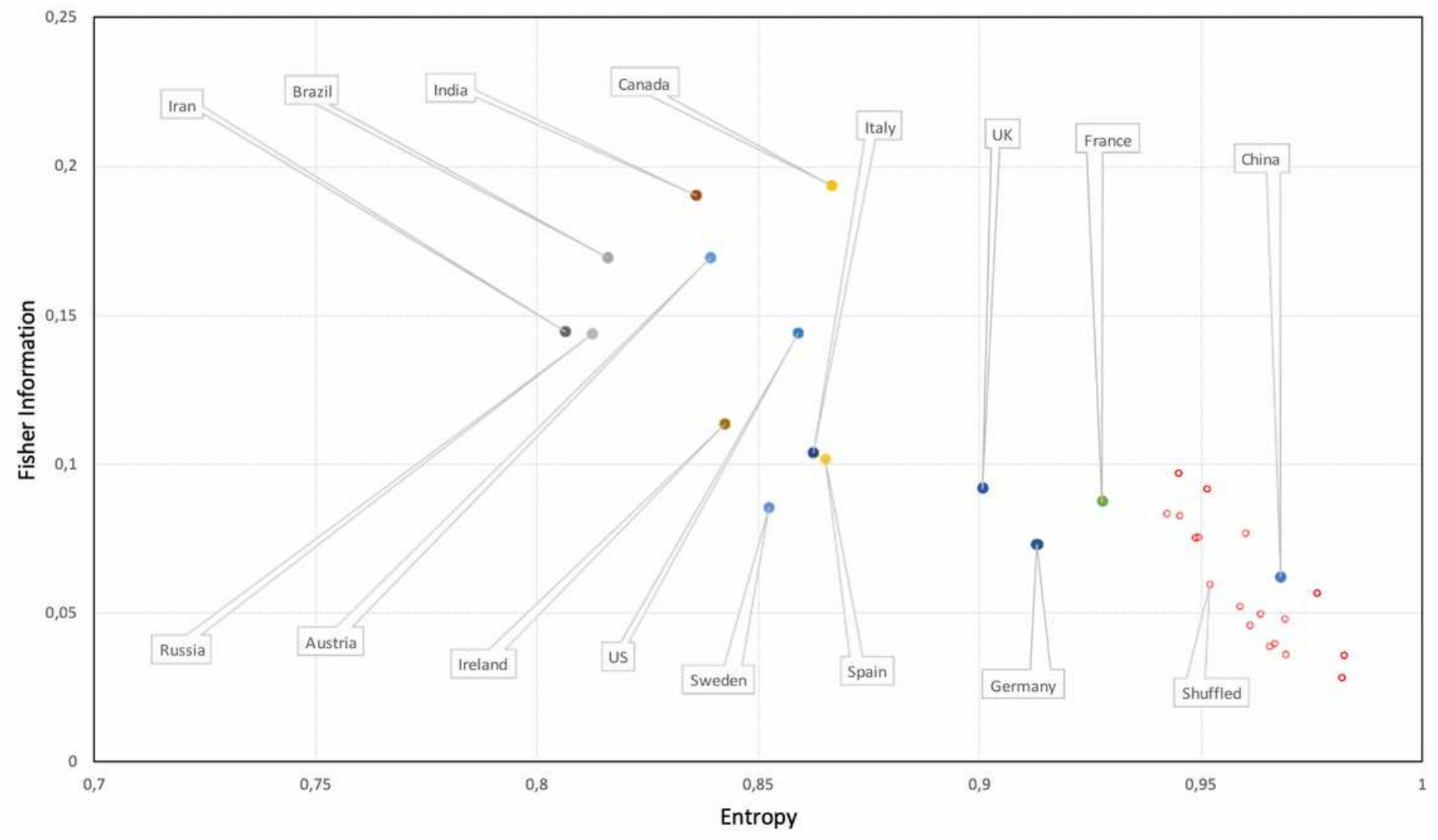

Figure 4

Illustration of the Shannon-Fisher causality plane ( $\mathrm{HxF})$ is applied to study the temporal evolution of the daily number of COVID-19 cases. For each time series of the number of daily incidences related to COVID19 , the permutation entropy measures are calculated considering $d=4$ to satisfy the common condition $T$ $>5 \mathrm{~d}$ !. 


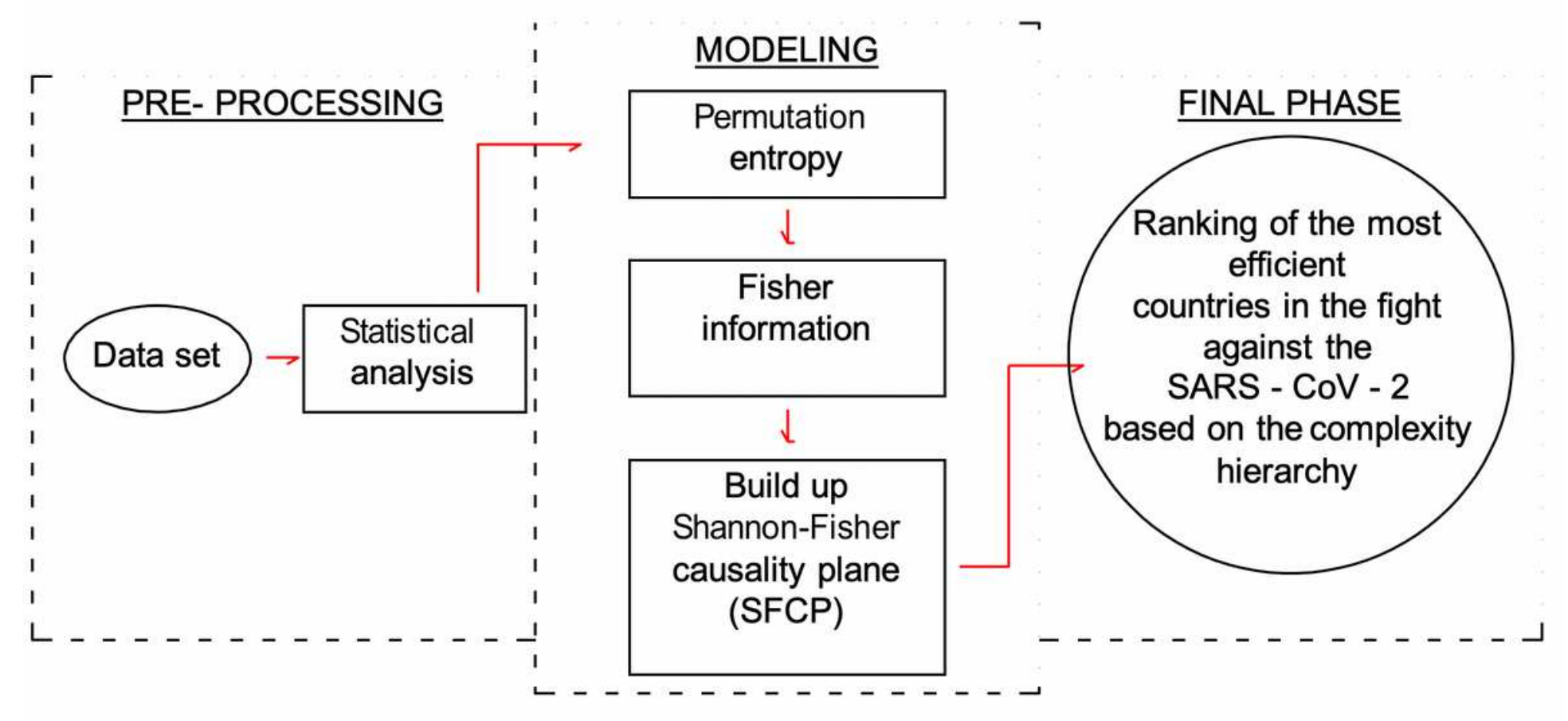

Figure 5

The flowchart of the theoretical framework of the procedures adopted in this research. Pre-processing corresponds to the analysis of data set and the application of statistical descriptive techniques. Modeling reflects the methods highlighted here and the final phase show the hierarchical complexity based on the Bandt \& Pompe permutation entropy H and Fisher Information F. 